\title{
Ultrafast dynamics of a magnetic antivortex: Micromagnetic simulations
}

\author{
Sebastian Gliga, Ming Yan, Riccardo Hertel, and Claus M. Schneider \\ Institut für Festkörperforschung IFF-9 “Elektronische Eigenschaften,” Forschungszentrum Jülich GmbH, D-52425 Jülich, Germany
}

(Received 30 November 2007; revised manuscript received 4 January 2008; published 29 February 2008)

\begin{abstract}
The antivortex is a fundamental magnetization structure which is the topological counterpart of the wellknown magnetic vortex. We study here the ultrafast dynamic behavior of an isolated antivortex in a patterned Permalloy thin-film element. Using micromagnetic simulations we predict that the antivortex response to an ultrashort external field pulse is characterized by the production of an additional antivortex as well as of a temporary vortex, followed by an annihilation process. These processes are complementary to the recently reported response of a vortex and, similar to the vortex, lead to the reversal of the orientation of the antivortex core region. In addition to its fundamental interest, this dynamic magnetization process could be used for the generation and propagation of spin waves for future logical circuits.
\end{abstract}

DOI: 10.1103/PhysRevB.77.060404

PACS number(s): 75.40.Gb, 75.40.Mg, 75.60.Jk, 75.75.+a

Extended ferromagnetic films often display complex magnetization patterns with a rich variety of features. ${ }^{1}$ This complexity can be reduced by decomposing the magnetic patterns into a few elementary magnetization structures, such as domains, domain walls, or vortices. The dynamic properties of such fundamental structures have been investigated thoroughly over the last years by isolating them in patterned elements. ${ }^{2}$ In particular, the magnetic vortex has attracted much interest. ${ }^{3-9}$ An equally fundamental, yet much less studied, magnetic structure is the antivortex, the topological counterpart of the vortex. In the complex structures occurring in extended soft-magnetic films, antivortices can be found almost as frequently as ordinary vortices: they occur in cross-tie domain walls, where they are enclosed by two adjacent vortex structures. ${ }^{10}$ While the in-plane magnetization distribution of an antivortex is very different from that of a vortex [see Fig. 1(a)], it contains, similar to the vortex, a tiny core $^{3}$ at its center in which the magnetization points perpendicular to the plane. Moreover, the two structures are related by underlying topological properties: In both cases, the local magnetization rotates by $360^{\circ}$ on a closed loop around the core. The structures differ by their opposite sense of rotation along such a loop, which is quantified by the winding number $w(w=-1$ for the antivortex, $w=+1$ for the vortex $) .11,12$ The winding number has been predicted to have a direct impact on the magnetization dynamics. ${ }^{11,13}$ However, not much is known to date about the dynamic properties of antivortices, even though several other fundamental magnetization structures have been analyzed in patterned thin-film elements. ${ }^{2}$ Studies with high spatial and temporal resolution have demonstrated that domains, domain walls, and vortices exhibit different excitation spectra. ${ }^{5,14}$ Such investigations have mostly focused on small perturbations and on reversible changes in these structures produced by an external field. ${ }^{15,16}$ However, in a recent study on cross-tie walls, Neudert et $a l .{ }^{17}$ have reported the creation of new cross ties, i.e., of vortex-antivortex pairs, in response to high-frequency magnetic fields. Also the field-pulse induced reversal of vortex cores has been found to be characterized by the creation of a vortex-antivortex pair. ${ }^{7-9}$ During this core switch, a transient cross-tie structure dissolves within a few tens of ps via an annihilation process. ${ }^{12,18}$ Using fully three-dimensional micromagnetic simulations we study here the ultrafast dynamic response of a single magnetic antivortex in response to external field pulses a few tens of ps long. We find that a rapid series of pair creation and annihilation processes occurs, which is complementary to the one observed in a vortex ${ }^{8}$ and which leads to the reversal of the antivortex core. This represents a fundamental process in magnetism on the nanoscale. The spin-wave bursts resulting from the annihilation process make the antivortex structure a possible source for spin waves to be used in logical circuits. ${ }^{19}$

Unlike vortices, it is rather difficult to isolate an antivortex, i.e., to prepare a nanostructure that contains only a single antivortex. This has been achieved by Shigeto et al. using thin-film elements consisting of four connected rings, ${ }^{20}$ where an antivortex was observed at the ring junction. Here we use a sample with a shape corresponding to the connecting part of four adjacent rings to stabilize an isolated antivortex [shown in Fig. 1(b)]. The sample is constructed from four circular segments with $200 \mathrm{~nm}$ radius. The four short stretching strips are $40 \mathrm{~nm}$ wide, and the thickness is $20 \mathrm{~nm}$. The in-plane shape anisotropy of this particular geometry can sustain an antivortex due to the tendency of the magnetization to align with the sample boundaries. The simulations have been performed with the micromagnetic finite-element code that we have used previously, e.g., in Ref. 8. The antivortex structure was obtained by selecting a suitable initial magnetic configuration and letting the structure relax. ${ }^{21}$ The material parameters used in the simulations are those of Permalloy: $A=13 \mathrm{pJ} / \mathrm{m}$ (exchange constant), $\mu_{0} M_{\mathrm{s}}=1.0 \mathrm{~T}$ (with $M_{s}$ the saturation magnetization), $K=0$ (anisotropy constant), and $K_{s}=10^{-4} \mathrm{~J} / \mathrm{m}^{2}$ (surface anisotropy). ${ }^{22}$ About 200000 tetrahedral elements were used for the discretization of the sample. This corresponds to a cell size of about $4 \mathrm{~nm}^{3}$. The magnetization dynamics was calculated using the LandauLifshitz-Gilbert equation with damping parameter $\alpha=0.01$. The isosurface representation introduced in Ref. 12 has been used to highlight the location of the antivortex core, that is, the region where the magnetization is exactly perpendicular to the sample plane $\left(m_{z}= \pm 1\right)$. This situation occurs where the $m_{x}=0$ and $m_{y}=0$ isosurfaces intersect ( $\mathbf{m}$ is the normalized magnetization, $\mathbf{m}=\mathbf{M} / M_{s}$ ).

The antivortex structure was perturbed by a short in-plane Gaussian-shaped pulse applied in the $y$ direction. In the case 

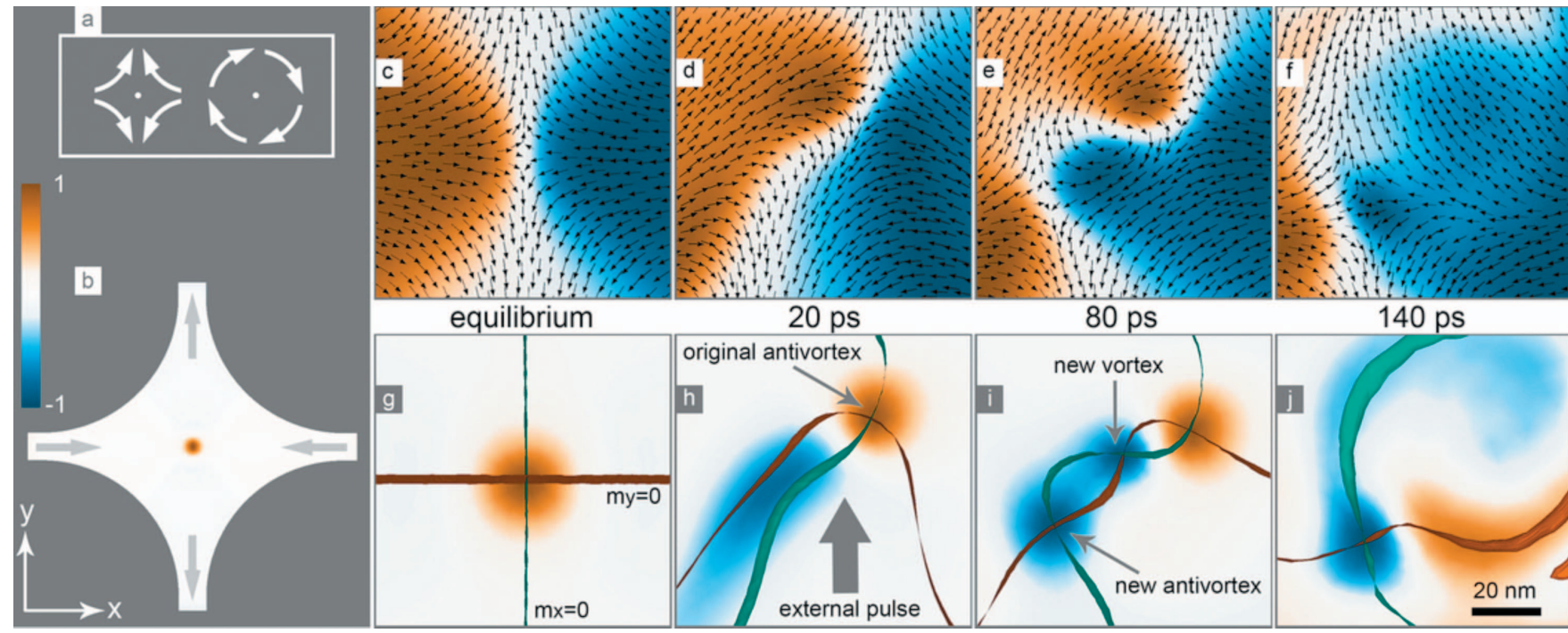

equilibrium
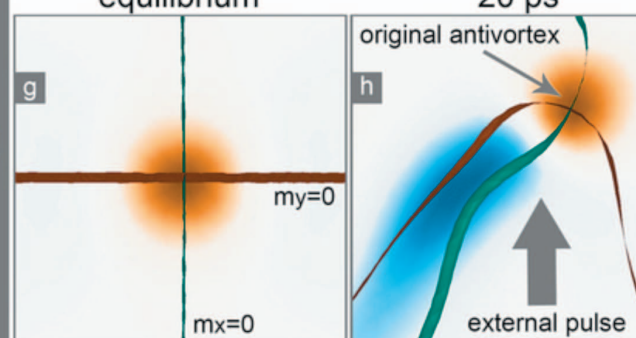

$80 \mathrm{ps}$

$140 \mathrm{ps}$

FIG. 1. (Color) (a) Schematic of an antivortex (left) compared with a vortex (right). The arrows represent the in-plane magnetization. (b) Modeled concave-shaped sample. The magnetization direction in the branches is represented by the grey arrows. The brown area at the center of the structure represents the out-of-plane component of the magnetization $m_{z}$, showing the antivortex core. [(c)-(j)] Distortion of the antivortex structure induced by an in-plane field pulse. The frames are snapshots at different times relative to the applied pulse's maximum. The arrows in the top row [(c)-(f)] represent the in-plane magnetization, while the colors represent the $x$ component of the magnetization $\left(m_{x}\right)$. In the bottom row $[(\mathrm{g})-(\mathrm{j})]$ the green and red "ribbons" are the $m_{x}=0$ and $m_{y}=0$ isosurfaces, respectively. The colors represent $m_{z}$.

shown in Fig. 1, the pulse had a maximum intensity of $60 \mathrm{mT}$ and a duration of $\sigma=80 \mathrm{ps}$. Figures $1(\mathrm{c})-1(\mathrm{j})$ show the magnetization dynamics in response to the applied field pulse. In the top row [Figs. 1(c)-1(f)], the in-plane magnetic structure is shown. The same structure is also shown in the bottom row [Figs. 1(g)-1(j)], but the colors there represent the perpendicular component of the magnetization $\left(m_{z}\right)$ and the crossing point of the isosurfaces helps display the position of the antivortex core. Following the application of the field pulse, the antivortex is displaced from its equilibrium position and the in-plane magnetization of the sample is distorted, Fig. 1(d). In a region close to the core, this distortion leads to the formation of a "dip" in which the magnetization rotates out of the plane in the direction opposite to the antivortex core, Fig. 1(h). The formation of such a dip in the case of an antivortex is analogous to the recently reported dip formation near a distorted vortex core..$^{8,23,24}$ Its direction is determined by the stray field of the antivortex core. ${ }^{25} \mathrm{Ap}$ proximately 80 ps after the pulse maximum, a new antivortex is emitted from the original antivortex, Fig. 1(e). However, according to the Poincaré-Hopf index theorem of vector fields, the total winding number of the magnetization in a thin-film element is a topological invariant, and the formation of a single antivortex would violate its conservation. A new vortex is thus also produced, which is located between the two antivortex structures. The newly formed antivortexvortex pair is made clearly visible by the two additional intersections of the isosurfaces, Fig. 1(i). This strongly inhomogeneous structure is eventually resolved through the annihilation of the initial antivortex with the newly created vortex, whose cores have opposite orientation. ${ }^{12}$ This results in a sudden reduction of the local exchange energy density and leaves behind the newly formed antivortex with oppositely magnetized core [Figs. 1(f) and 1(j)].
To the best of our knowledge, the production of an antivortex-vortex pair from an antivortex structure is a previously unreported micromagnetic process. The resulting transient magnetic configuration could be called an anti cross-tie wall, i.e., a single vortex enclosed by two antivortices, which has never been observed in a stable magnetic structure. It is also remarkable that, in spite of the very different magnetic in-plane structure of vortices and antivortices, their ultrafast dynamics are analogous, involving the creation of new magnetic structures followed by a destruction process. Here the strength of the isosurface representation is obvious: it captures the main features of the processes unfolding in both vortex and antivortex structures,${ }^{26}$ demonstrating the complementarity between them.

In the case shown in Fig. 1, the system eventually relaxes back to an antivortex structure. However, the stability of an isolated antivortex is rather weak compared to that of a vortex. This is due to the magnetic flux, which in an antivortex has a saddle-point configuration while the (energetically favorable) closure of the flux is obtained around an ordinary vortex. The magnetic configuration in the sample we have used leads in fact to the formation of magnetic surface and volume charges, which reduce the stability of the antivortex. ${ }^{27}$ This easily introduces the possibility of a vortex nucleating at the sample boundaries if stronger external field pulses are applied. Using isosurfaces, the vortex nucleation process at our sample's boundaries can easily be described and visualized. The behavior of the $m_{x}=0$ and $m_{y}=0$ isosurfaces is shown in Fig. 2 for two field pulse strengths. The crossing of the isosurfaces at the boundaries indicates that a vortex is nucleated. ${ }^{28}$ The introduction of a vortex in the sample affects the dynamics of the antivortex and thus also the final equilibrium structure. The different possible final states are shown in Fig. 3 as a function of the applied pulse's 


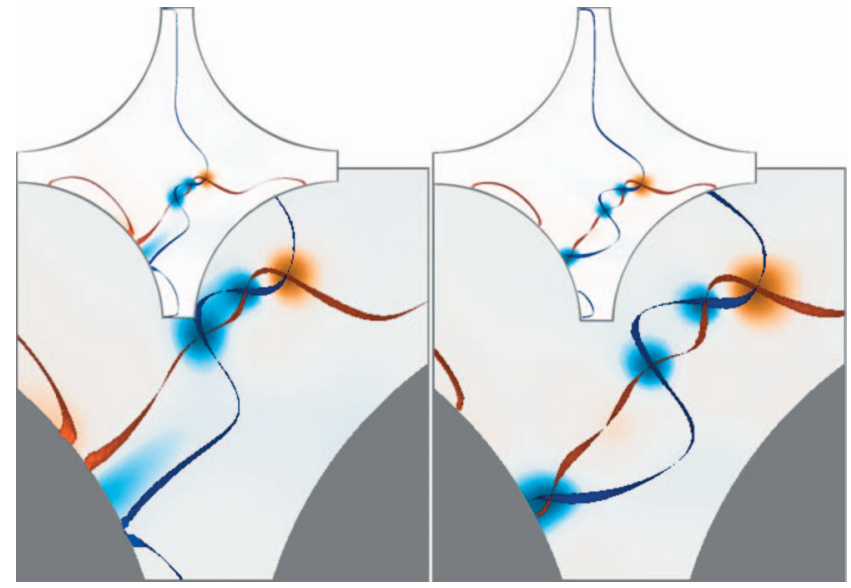

FIG. 2. (Color) Vortex nucleation at the sample edge during the pair creation process. Left: For a Gaussian pulse of $60 \mathrm{mT}$ and 80 ps duration, no edge nucleation occurs. Right: For a stronger pulse $(80 \mathrm{mT})$ of the same duration, the $m_{x}$ and $m_{y}$ isosurfaces cross at the sample boundary, indicating the nucleation of a vortex.

strength and duration. It indicates that while it is rather difficult to isolate an antivortex, it is pretty easy to dissolve it. Starting with low and short field pulses, there is a set of parameters for which the system is slightly excited, causing the antivortex core to rotate about its equilibrium position (region $A$ in Fig. 3). The modes excited by such low field pulses have been investigated in Ref. 15 . When pair production and, consequently, a core switch is induced, the studied system relaxes into an antivortex configuration only for a rather narrow set of field pulse parameters (region $B$ ). For stronger or longer pulses, vortex nucleation occurs concurrently to the antivortex core switch, as shown in Fig. 2. The vortex can then migrate through the sample, leading to the expulsion of the antivortex after a few ns (region $C$ ). In region $D$ (for pulses above $60 \mathrm{mT}$ ), the pulse is sufficiently strong to induce the production of new pairs originating from both the antivortex and the nucleated vortex. The result is the

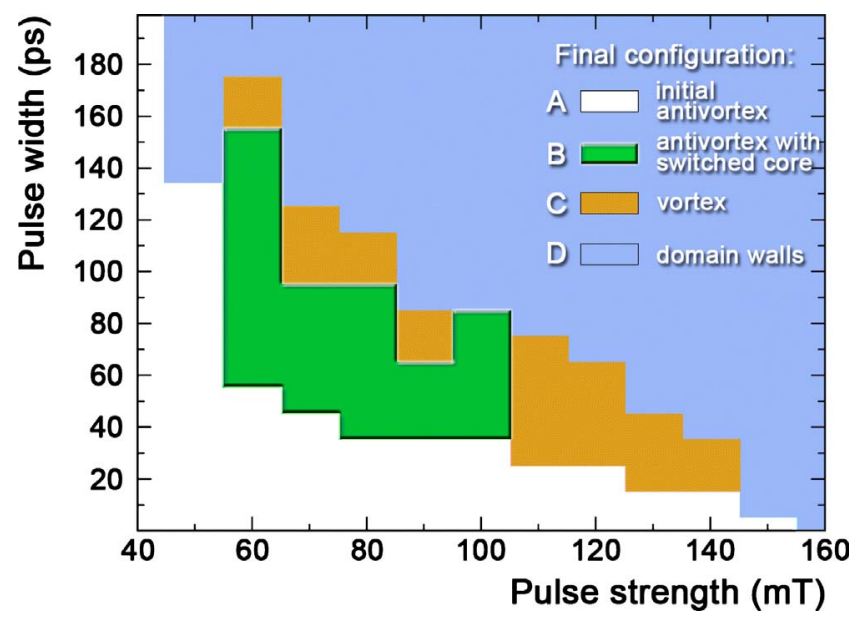

FIG. 3. (Color online) Final magnetic configurations depending on the field pulse strength and duration. The duration is defined as the width of the Gaussian. The field was varied in increments of $10 \mathrm{ps}$ and for every $10 \mathrm{mT}$.

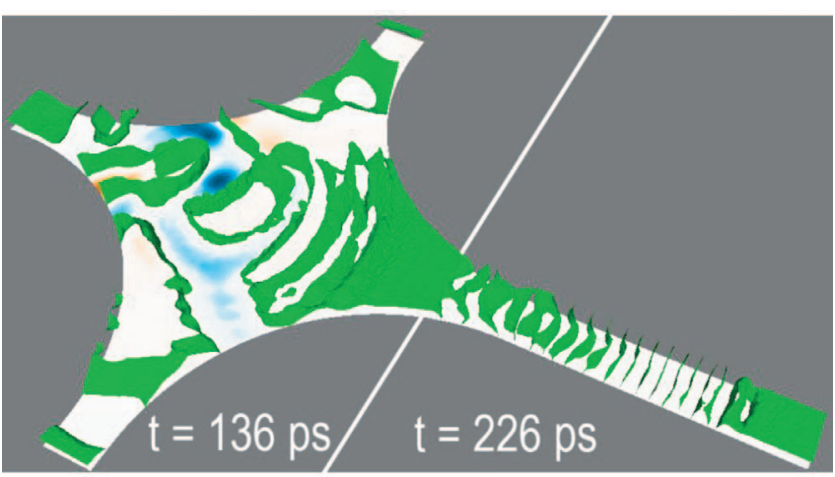

FIG. 4. (Color) Spin-wave propagation at $136 \mathrm{ps}$ (left) and 226 ps (right) after the pulse's maximum. The spin waves generated by the core reversal (left) shortly later propagate smoothly into the branch (right). The $m_{z}=0$ isosurfaces shown in green allow to visualize the wave fronts. The blue spot represents the switched core $\left(m_{z}\right)$ using the same scale as in Fig. 1.

generation of a transient state containing multiple cross ties. A series of annihilations ${ }^{12,18,29}$ occurs within a few ten ps, leading to a final magnetic configuration consisting only of a pair of head-to-head and tail-to-tail domain walls.

The annihilation of a vortex and an antivortex with opposite core magnetization has been shown to be connected with the emission of spin-wave bursts in the $\mathrm{GHz}$ range. ${ }^{12,18}$ It has therefore been suggested that the core switch mechanism of a vortex could be used to inject spin waves into a strip acting as a waveguide. ${ }^{30}$ The spin waves could then be processed in logical circuits. ${ }^{19}$ In this context, the antivortex structure could equally be used as a spin-wave source. If its stability can be increased, an antivortex would probably be more suitable than a vortex for this purpose: When a disk-shaped source is used (in the case of the vortex core switch) a $90^{\circ}$ Néel wall is inevitably present between the source and the strip along which the waves propagate. Such domain walls have been reported to have unfavorable properties concerning spin wave propagation, mostly reflecting the spin waves. ${ }^{31}$ In contrast, as shown in Fig. 4, the spin waves produced in the antivortex can propagate unhindered into a branch, which naturally extends the magnetic configuration of the astroid-like sample. All branches can be extended to connect to other astroid-like elements. Such a network could constitute an antidot array ${ }^{32}$ and provide a regular lattice of antivortices.

What does the study of antivortex dynamics lead to? First, the complex and ultrafast modifications that an antivortex can undergo by applying a short field pulse are of fundamental interest. A short field pulse can be used to switch the core of an antivortex. This occurs through the rapid creation of a new antivortex and the annihilation of the original one with a short-lived vortex. Second, we find that the static topological complementarity of vortices and antivortices is equally exhibited in their dynamical behavior. The dynamics of antivortices deserves further investigation since it likely plays an equally important role as the dynamics of vortices in the case of pulse-induced cross-tie domain-wall transformations. ${ }^{17}$ In this context it will be necessary to determine whether the pair-creation reported in Ref. 17 is directly due to the dy- 
namics described above, since larger distances of a few hundreds of $\mathrm{nm}$ are involved in cross-tie walls. Third, the annihilation of transient magnetic structures during the antivortex core switching process could be used, e.g., for generating spin waves for logical circuits. ${ }^{19}$
${ }^{1}$ A. Hubert and R. Schäfer, Magnetic Domains-The Analysis of Magnetic Microstructures (Springer, Berlin, 1998).

${ }^{2}$ Spin Dynamics in Confined Magnetic Structures III, edited by B. Hillebrands and A. Thiaville, vol. 101 of Topics in Applied Physics No. 101 (Springer, Berlin, 2006).

${ }^{3}$ A. Wachowiak, J. Wiebe, M. Bode, O. Pietzsch, M. Morgenstern, and R. Wiesendanger, Science 298, 577 (2002).

${ }^{4}$ V. Novosad, M. Grimsditch, K. Y. Guslienko, P. Vavassori, Y. Otani, and S. D. Bader, Phys. Rev. B 66, 052407 (2002).

${ }^{5}$ J. P. Park, P. Eames, D. M. Engebretson, J. Berezovsky, and P. A. Crowell, Phys. Rev. B 67, 020403(R) (2003).

${ }^{6}$ S. B. Choe, Y. Acremann, A. Scholl, A. Bauer, A. Doran, J. Stohr, and H. A. Padmore, Science 304, 420 (2004).

${ }^{7}$ B. Van Waeyenberge et al., Nature (London) 444, 461 (2006).

${ }^{8}$ R. Hertel, S. Gliga, M. Fähnle, and C. M. Schneider, Phys. Rev. Lett. 98, 117201 (2007).

${ }^{9}$ Q. F. Xiao, J. Rudge, B. C. Choi, Y. K. Hong, and G. Donohoe, Appl. Phys. Lett. 89, 262507 (2006).

${ }^{10}$ E. E. Huber, Jr., D. O. Smith, and J. B. Goodenough, J. Appl. Phys. 29, 294 (1958).

${ }^{11}$ A. P. Malozemoff and J. C. Slonczewski, Magnetic Domain Walls in Bubble Materials (Academic Press, New York, 1979).

${ }^{12}$ R. Hertel and C. M. Schneider, Phys. Rev. Lett. 97, 177202 (2006).

${ }^{13}$ A. A. Thiele, Phys. Rev. Lett. 30, 230 (1973).

${ }^{14}$ J. Raabe, C. Quitmann, C. H. Back, F. Nolting, S. Johnson, and C. Buehler, Phys. Rev. Lett. 94, 217204 (2005).

${ }^{15}$ H. Wang and C. E. Campbell, Phys. Rev. B 76, 220407(R) (2007).

${ }^{16}$ K. Kuepper, M. Buess, J. Raabe, C. Quitmann, and J. Fassbender, Phys. Rev. Lett. 99, 167202 (2007).

${ }^{17}$ A. Neudert, J. McCord, R. Schäfer, and L. Schultz, Phys. Rev. B 75, 172404 (2007).

${ }^{18}$ K.-S. Lee, S. Choi, and S.-K. Kim, Appl. Phys. Lett. 87, 192502 (2005).

${ }^{19}$ R. Hertel, W. Wulfhekel, and J. Kirschner, Phys. Rev. Lett. 93,
257202 (2004)

${ }^{20}$ K. Shigeto, T. Okuno, K. Mibu, T. Shinjo, and T. Ono, Appl. Phys. Lett. 80, 4190 (2002).

${ }^{21}$ The antivortex structure is not the only stable magnetization state here. Using dynamic relaxation methods, we have equally observed the formation of a metastable vortex state and a state containing two domain walls.

${ }^{22}$ J. O. Rantschler, P. J. Chen, A. S. Arrott, R. D. McMichael, W. F. Egelhoff, and B. B. Maranville, J. Appl. Phys. 97, 10J113 (2005).

${ }^{23}$ V. Novosad, F. Y. Fradin, P. E. Roy, K. Buchanan, K. Y. Guslienko, and S. D. Bader, Phys. Rev. B 72, 024455 (2005).

${ }^{24}$ S. Kasai, Y. Nakatani, K. Kobayashi, H. Kohno, and T. Ono, Phys. Rev. Lett. 97, 107204 (2006).

${ }^{25}$ S. Gliga, R. Hertel, and C. M. Schneider, Physica B 403, 334 (2008).

${ }^{26}$ The isosurface representation of the antivortex core switching is almost identical to the isosurface representation of the vortex core switching, cf. Fig. 2 of Ref. 8

${ }^{27}$ S. Gliga, R. Hertel, and C. M. Schneider (unpublished).

${ }^{28}$ The isosurfaces are perpendicular to the sample edges, which is a result of the Brown boundary condition $\left.\boldsymbol{\nabla} m_{i}\right|_{\partial \Omega} \cdot \mathbf{n}=0 \quad(i$ $=x, y, z)$, where $\mathbf{n}$ is the unit vector normal to the surface $\partial \Omega$. The magnetization gradient is therefore nonvanishing only along a direction parallel to the sample edges. As isosurfaces and gradient lines are perpendicular to each other, the $m_{x}=0$ and $m_{y}$ $=0$ isosurfaces are always perpendicular to the sample edges.

${ }^{29}$ K.-S. Lee, B.-W. Kang, Y.-S. Yu, and S.-K. Kim, Appl. Phys. Lett. 85, 1568 (2005).

${ }^{30}$ S. Choi, K.-S. Lee, K. Y. Guslienko, and S.-K. Kim, Phys. Rev. Lett. 98, 087205 (2007).

${ }^{31}$ M. Buess, J. Raabe, K. Perzlmaier, C. H. Back, and C. Quitmann, Phys. Rev. B 74, 100404(R) (2006).

${ }^{32}$ L. J. Heyderman, S. Czekaj, F. Nolting, D.-H. Kim, and P. Fischer, J. Magn. Magn. Mater. 316, 99 (2007). 